rotated every $4 \mathrm{hr}$. Only 38.6\% was trained (control of precaution and protection) by the Ministry of Public Health. In one year later, The worker had an accident or injury (punch with needle or other sharp) from IW during working 42.5\%, 37.8\% have had contaminated or touch with the infectious fluid, $18.9 \%$ had traffic accident (car turnover, clash, offside cone) and 8.3\% had the IW or leachates flood pour out from vehicle or container. The factors associated with risk of the health of the IW worker taken into account of affect of other factors, it were found that prevention and precaution training, job position, age and yearly health check were statistical significant with the health risk, it were found that prevention and precaution training $(\mathrm{OR}=4.61)$ job position $(\mathrm{OR}=3.68)$, Age $(\mathrm{OR}=2.97)$ and yearly health check $(\mathrm{OR}=0.96)$ were statistical significant with the health risk. The problems of IW transportation from hospital by PTS were health risk. The infectious waste management (IWM) which organised by PTS need to develop for better system and quality.

\section{HEALTHCARE WORKERS AND OCCUPATIONAL EXPOSURE TO ANTINEOPLASTIC DRUGS IN ONCOLOGY WARDS}

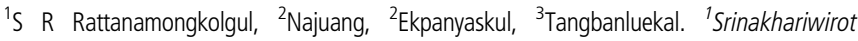
University, Nakhon Nayok, Thailand;' ${ }^{2}$ Faculty of Medicine, Srinakharinwirot Univesity, Nakhon Nayok, Thailand; ${ }^{3}$ Faculty of Medicine. Siriraj Hospital, Mahidol University, Bangkok, Thailand

\subsection{6/oemed-2013-101717.172}

Objective To quantify levels of Ifosfamide (IF) in urine among healthcare workers in a hospital setting.

Methods This cross-sectional study identified 70 subjects who handled IF in oncology wards or antineoplastic drug preparing units at a medical school hospital. Their urine samples were collected at 1-2 hours prior to the end of each work shift, the levels of IF was determinate by gas chromatography mass spectrometer (GC/MS). Information on demographic information, work practice, work position, working station, medication timing and dosages including the time of urine collections was collected using self-administered questionnaires and personal interviews.

Results IF levels were detected in urine of 20 personnel $(28.6 \%$ of total) with geometric mean of $3.6 \mathrm{ng} / \mathrm{mL}$, arithmetic mean $43.6 \mathrm{ng} / \mathrm{mL}$, min-max of $16-526 \mathrm{ng} / \mathrm{mL}$. Among IF-positive subjects, $53.8 \%$ of workers with were in the age group of 41-60 years. Levels of IF were found in all subjects who were drug-preparing workers were found positive tests for IF. The levels of urine IF (geometric mean) were found to be of $526 \mathrm{ng} / \mathrm{mL}$ among the pharmacist assistants, $3.1 \mathrm{ng} / \mathrm{mL}$ for cleaning worker, $2.9 \mathrm{ng} / \mathrm{mL}$ for nurses, and $86.8 \mathrm{ng} / \mathrm{mL}$ for pharmacists.

Conclusions All groups of healthcare workers in the processes of anti-neoplastic medication especially among pharmacist assistants and cleaning workers. Although in a high standard protection against the oncological chemicals, health personnel are at risk of contaminations especially among professional assistants and general workers.

\section{POSSIBLE HIV EXPOSURE: LABORATROY WORKERS KNOWLEDGE ATTITUDES AND BEHAVIOUR}

K S Wilson, Zungu, Nattey. NIOH, Johannesburg, South Africa

10.1136/oemed-2013-101717.173
A company wide KAP survey was conducted around HIV, but in this workplace, exposure to HIV through work exposures was also possible. In a high prevalence HIV country workers perceptions and behaviour around needlesticks play a role in the occupational health and safety

Methods This survey was nested within the larger KAP survey which was distributed to all employees of the company with their pay slips. The questions covered knowledge of correct procedures to follow, likely hood to take prophylaxis and complete the course.

Results 1497 employees participated in the KAP survey which covered all regions of South Africa and represented all occupations within the company. One third of participants were only partly aware or not al all aware of the correct procedure to follow after a needlestick, and those who were unfamiliar wit the procedure were also more likely to over estimate the ideal window for starting post exposure prophylaxis. 33\% of participants would only take PEP if the injury warranted it. These findings were significantly associated with education but not job category. Age also played a significant role in the perception of risk and compliance. Only $9 \%$ of staff reported a needlestick in the past 5 years but in a high prevalence HIV country these people are at high risk.

Conclusions The training of laboratory staff needs to be undertaken with focus on those with lower education levels to ensure understanding. This will allow all employees to have the best information around needlesticks and post exposure prophylaxis.

\section{EXPOSURE TO STATIC MAGNETIC FIELDS INCREASES RISK OF ACCIDENTS AMONG WORKERS FROM A MEDICAL IMAGING DEVICE PRODUCTION FACILITY}

S I M Bongers, Slottje, Portengen, Kromhout. IRAS, Utrecht University, Utrecht, Nederland

\subsection{6/oemed-2013-101717.174}

Acute neurocognitive and balance effects, and reported health complaints such as vertigo related to static magnetic fields (SMF) from magnetic resonance imaging (MRI) devices were the reason to study the effects of these exposures on the occurrence of accidents. In this first retrospective cohort study among workers from a facility producing medical imaging devices we studied the association between SMF and occurrence of accidents in general and during commuting.

Methods SMF exposure was assessed with a manufacturing facility specific historical job exposure matrix linked to company records on individual job histories, resulting in two SMF exposure measures: exposure in the year of a self-reported accident (recent exposure) and cumulative exposure up to the end of the year an accident was reported (career exposure). Data on occurrence of accidents was collected through an online questionnaire (1479 participants, participation rate 30\%). Data on injuries and physician-treated injuries due to accidents in the past 12 months were analysed with logistic regression. Discrete-time survival analysis was applied to historic data on the reported year of the first ever (near) traffic accident during commute to and from work.

Results High recent SMF exposure was found to be related to an increased risk of accidents leading to injury (Odds Ratio 4.39, 95\% Confidence Interval 1.20-16.06) with a stronger effect for injuries treated by a physician (OR 6.28, 95\%CI 1.7023.26). High recent SMF exposure was associated with increased risk (Hazard Rate 3.21, 95\% Confidence Interval 1.36-7.18) of (near) accidents during commute from home to work, but not from work. No increased risks were found with respect to career exposure after adjustment for recent exposure. 
Conclusion This retrospective cohort study among workers exposed to MRI-related SMF showed that recent SMF exposure is associated with increased risks of accidents resulting in injuries and (near) traffic accidents during commute from home to work.

\section{Session: W. Methods}

\section{CLINICAL RECORD KEEPING QUALITY INDICATORS FOR OCCUPATIONAL HEALTH SERVICES}

${ }^{1}$ R R Stilz, ${ }^{2}$ Madan. 'Plumstead, United Kingdom; 'Guy's and St Thomas' NHS Foundation Trust, London, United Kingdom

\subsection{6/oemed-2013-101717.175}

Objectives In any clinical speciality, the collection of accurate clinical information is a pre-requisite to providing high quality clinical care. Structured medical records can improve measures of performance and outcome in clinical care. In the United Kingdom (UK) record keeping standards have been developed for hospital medical records but none are available for occupational health services (OHS). This project reviewed the available evidence to develop generally applicable record keeping quality indicators (QI) for OHS.

Methods The results of three comprehensive UK literature reviews on record keeping standards were examined. Studies with direct or indirect relevance to the OHS setting were included. Evidence statements on medical record keeping were extracted. Bias, outcome measures and OHS implications were evaluated. Candidate QI were mapped against the results of a two-stage national consensus exercise on record standards, and suitable QI were developed.

Results 84 publications were included in the review, 22 were selected for full text appraisal. Performance improvements were shown with general structured assessment forms in seven publications and with context specific forms in 12 publications. No specific studies based in OHS were retrieved; however one publication was relevant to occupational health care. Following critical appraisal of the studies and the mapping process, four generally applicable record keeping QIs, with the headings "findings", "treatment", "assessment, and "advice" were developed. Conclusions General and context specific record keeping standards have been shown to improve the quality of care in other medical specialities. We have developed four general record keeping QI for use in OHS. These indicators are applicable across all employment sectors. The indicators will be incorporated into a national UK clinical registry for OHS. Further work will be done to validate these QI in practice. Context specific record keeping indicators should be developed in occupational medicine.

\section{ANALYSIS OF HIERARCHICAL DATA; COMPARING META- ANALYTICAL TO MULTILEVEL ESTIMATES}

${ }^{1} \mathrm{G} N$ Ntani, ${ }^{2} \mathrm{H}$ I Inskip, ${ }^{2} \mathrm{D} \mathrm{C}$ Coggon. ${ }^{1} M R C$, Lifecourse Epidemiology Unit, Southhampton, United Kingdom; ${ }^{2}$ MRC, Southampton, United Kingdom

\subsection{6/oemed-2013-101717.176}

Objectives To compare pooled risk estimates obtained by metaanalytical methods with the corresponding risk estimates derived by analysis of individual data, using hierarchical methods, and to explore explanations for possible differences.

Methods The data were obtained from a study of 12,426 participants from 47 occupational groups (mostly nurses and office workers) in 18 countries. Pain in the low back and wrist/hand that interfered with everyday activities and exposure to possible risk factors had been collected using standardised questionnaires. Unadjusted associations with potential risk factors were explored using logistic regression separately for each occupational group and then the 47 risk estimates for each risk factor were synthesised in a meta-analytical model. Risk estimates were also obtained from the individual data using multilevel logistic models. The multi-level estimates were then compared with the corresponding pooled estimates derived from the meta-analysis.

Results For most risk factors, the odds ratios were similar using the two methods, though the confidence intervals for the oddsratio estimates obtained from the meta-analysis model were wider than the confidence intervals for those derived from the multilevel model. When disabling low back pain was used as an outcome, the mean of the ratios of the odds ratios derived from multilevel modelling to those derived from meta-analysis was 0.99 (range 0.87 to 1.07 ). When disabling wrist/hand pain was taken as the outcome, the mean ratio was 0.97 (range 0.84 to 1.11).

Conclusions In the analysis of these data, pooled risk estimates obtained by meta-analysis were very similar to those derived from multilevel analysis of the individual data. However, circumstances in which estimates from the two methods may differ will be discussed.

\section{USE OF PROPENSITY SCORES IN OCCUPATIONAL HEALTH?}

${ }^{1}$ A D Descatha, ${ }^{2}$ Leclerc, ${ }^{3}$ Herquelot. ${ }^{1}$ Garches, France; ${ }^{2}$ Inserm, Villejuif, France; ${ }^{3}$ UVSQ Inserm, Villejuif, France

\subsection{6/oemed-2013-101717.177}

Objectives Intervention studies are needed in occupational health in order to test if work improvements are effective, although not always possible. In the last decade, propensity score analysis has been widely used in clinical settings for treatments for which randomised controlled trials are unlikely to be conducted, such as socioeconomic factors in public health. It gives an estimate of a treatment effect in making treated and untreated group comparable for confounding factors by modelling treatment probability. We aimed to describe the frequency of propensity score cited in occupational health literature and its use.

Methods Using the key words "occupational" and "propensity score", studies were selected from PubMed database (last 20 years). Frequency of citation was compared to two other requests "clinical" "propensity score" - "public health" not "occupational" "propensity score". Embase and Web of sciences were also used to find extra papers. Relevant information was extracted including use of propensity score.

Results Twenty-four papers were found in PubMed with propensity score in occupational health $[0.01 \%$ of all citations of this area, versus $1210(0.06 \%)$, and $2731(0.07 \%)$ in clinical or other public health domains respectively $\mathrm{P}<0.001$ ]. Among papers in occupational health, 12 were relevant, published since 2005 , mostly from US teams $(\mathrm{n}=10)$. The analyses included many subjects (median 7314), coming from pre-existing databases. The studies dealt with treatment evaluation $(n=2)$, economic evaluation $(\mathrm{n}=2)$, and risk factors analysis $(\mathrm{n}=5)$. Three papers studied implementation of programs in occupational setting, two for economic or quality purpose, one for effectiveness of multidisciplinary primary prevention program including work adaptation. 